Editorial

\title{
Pediatric EEG and the COVID-19 Pandemic: Can We Develop a Safe Transition Pathway to Reopen Our Laboratories?-Phase 2
}

\author{
Jorge Vidaurre $^{1}$ Monica P. Islam ${ }^{1} \quad$ John R. Mytinger ${ }^{1}$ \\ ${ }^{1}$ Division of Pediatric Neurology, Department of Pediatrics, \\ Nationwide Children's Hospital, Columbus, Ohio, United States
}

J Pediatr Epilepsy 2021;10:1-2.

We previously reviewed our strategy to reopen our electroencephalography (EEG) laboratory at Nationwide Children's Hospital following a period of near closure during the COVID19 pandemic. $^{1}$ Between March 2020 and May 2020, only emergent and urgent EEGs were completed with the recognition that prolonged postponement of nonurgent EEGs had potential to negatively affect patient care. Over 400 open EEG orders were evaluated for urgency and mostly postponed. The evolution of state- and hospital-based recommendations, including the gradual return to performing nonurgent procedures, has necessitated a modification of our EEG laboratory's disease mitigation strategies. We present those modifications here. The safety of our technicians, patients, caregivers, and community remain paramount in our recommendations.

Scheduling/preprocedure considerations:

1. As of this writing, preprocedure COVID-19 testing by polymerase chain reaction (PCR) is not a requirement to perform outpatient routine EEGs. In the early days of the pandemic restrictions, we were fortunate to have the capability to perform preprocedure COVID-19 testing by PCR within 24 hours prior to nonemergent EEGs. This was scheduled for all children (but not the accompanying adult) undergoing the study and results were available prior to the patient coming to the laboratory. Only patients with negative test results completed nonurgent studies. However, given the low rate of positivity in this asymptomatically screened population (based on local institutional data), and the need to conserve testing supplies for inpatient admissions, we currently do not perform COVID-19 pretesting in asymptomatic children.

2. Screening questions include whether the patient or any household member has been positive for COVID-19 in the past 30 days and whether the patient or any household member is under quarantine for an exposure. Also, the scheduler asks whether the patient has signs and symptoms of current or recent illness. These questions are reviewed at the time of scheduling and in the days prior to the test. Children referred for a nonurgent EEG who have been recently COVID-19 positive, had a recent COVID-19 exposure, or have a current upper respiratory infection of unknown etiology are asked to reschedule following an appropriate period of quarantine or isolation based on the Center for Disease Control and Prevention guidelines. ${ }^{2,3}$

3. To reduce number of individuals in the registration area, our scheduling volume reopened with $25 \%$ of the prepandemic capacity. We have now returned to a full schedule with ongoing mitigation measures and monitoring.

Testing/procedure considerations:

1. We continue to permit only a single accompanying adult.

2. Employees are asked to check their temperature every day and monitor for signs of COVID-19-related illness. Prior to entering the EEG laboratory, patients and visitors are screened with temperature checks at locations adjacent to inpatient space.

3. In an effort to minimize the time spent in common areas such as the waiting room, the child and accompanying adult are required to wait in the car or outside the laboratory until the room is ready and available. ${ }^{4}$

4. We continue to implement technicians' use of personal protective equipment in the form of surgical mask, gloves, and goggles.

5. We continue to require mask wearing by children 2 years and older (developmentally capable) and the accompanying adult. The institution provides a surgical mask to all visitors to our facilities.

6. We continue hyperventilation precautions. Our previous guidelines recommended performance of hyperventilation only in children with known absence seizures or a history concerning for absence seizures. Again, given the low rate of COVID-19 positivity of asymptomatic children at our center, hyperventilation is now performed in all children (who are without contraindications). Children
Address for correspondence Jorge Vidaurre, MD, Division of Pediatric Neurology, Department of Pediatrics, Nationwide Children's Hospital, Columbus, Ohio, United States

(e-mail: jorge.vidaurre@nationwidechildrens.org).
C 2020. Thieme. All rights reserved. Georg Thieme Verlag KG,

Rüdigerstraße 14 ,

70469 Stuttgart, Germany
DOI https://doi.org/ 10.1055/s-0040-1721436. ISSN 2146-457X. 
perform hyperventilation while wearing a mask. Transient unmasking is permitted only in children who are uncooperative or do not tolerate the mask. Our protocol includes caregiver-guided hyperventilation with physical distancing of the EEG technician.

\section{Posttesting/postprocedure considerations:}

1. Room surfaces and equipment in contact with patients are cleaned after every use with approved germicides.

Communication among technicians, families, neurologists, and primary care providers in this changing environment remains vital as community isolation measures have relaxed even since the laboratory initially reopened. Precautions for individual testing scenarios during scheduling and testing will continue to be considered relative to local patterns of COVID-19 spread. As before, vigilance to the changing public health situation will guide further modification of this proposed guideline.

\section{Conflict of Interest}

None declared.

\section{References}

1 Vidaurre J, Islam MP, Mytinger JR. Pediatric EEG and the COVID-19 pandemic: can we develop a safe transition pathway to reopen our laboratories? J Pediatr Epilepsy 2020;2(09): $29-30$

2 Information for Pediatric Healthcare Providers. Available at: https://www.cdc.gov/coronavirus/2019-ncov/hcp/pediatric-hcp. html. Accessed October 14, 2020

3 When to Quarantine. Available at: https://www.cdc.gov/coronavirus/2019-ncov/if-you-are-sick/quarantine.html. Accessed October 14, 2020

4 Chu DK, Akl EA, Duda S, Solo K, Yaacoub S, Schünemann HJ. COVID-19 Systematic Urgent Review Group Effort (SURGE) study authors. Physical distancing, face masks, and eye protection to prevent person-to-person transmission of SARS-CoV-2 and COVID-19: a systematic review and meta-analysis. Lancet 2020; 395(10242):1973-1987 